Erschienen in: Harras, Gisela (Hrsg.): Die Ordnung der Wörter. Kognitive und lexikalische Strukturen. Jahrbuch 1993 des Instituts für deutsche Sprache. Berlin/New York: de Gruyter, 1995. S. 328-354.

(Jahrbuch des Instituts für deutsche Sprache 1993)

\title{
EDELTRAUD WINKLER
}

\section{Die Darstellbarkeit lexikalischen Wissens - am Bei- spiel kommunikativer Ausdrücke des Deutschen}

\section{Vorbemerkung}

Wie bereits der Titel umreiBt, soll hier ausführlicher behandelt werden, auf welche Weise man lexikalisches Wissen für einen bestimmten Bereich der Sprache repräsentieren kann. Im vorliegenden Fall handelt es sich um die kommunikativen Ausdrücke des Deutschen, für die eine mögliche Darstellungsform vorgestellt wird. ${ }^{1}$ Mit ihrer Hilfe wird versucht, alle Aspekte des lexikalischen Wissens über kommunikative Ausdrücke - die ich im folgenden der Einfachheit halber auch Sprechaktverben nennen werde - in systematischer Weise darzustellen. Dabei werden Situationstypen für sprachliches Handeln beschrieben, die sowohl die Bedingungen für das sprachliche Handeln überhaupt enthalten als auch typische $\mathrm{Ka}$ tegorien von Kommunikation beschreiben. So orientiert sich die Darstellungsweise an einer möglichst vollständigen Erfassung der Gebrauchsbedingungen für die einzelnen Sprechaktverben, wodurch beispielsweise die Möglichkeit eröffnet wird, auf einer anderen Ebene neu in die Diskussion über eine Gebrauchsgrammatik einzutreten, nur um einen Aspekt herauszugreifen. Ein anderer tangiert die Auseinandersetzung darüber, welcher Art das konzeptuelle Wissen, die konzeptuellen Strukturen sind, die hier relevant sind. Es soll gewissermaßen eine Art von 'szenischem Wissen' vermittelt werden, das mit der Verwendung des einzelnen Sprechaktverbs abgerufen wird. Auf diese Weise könnte eine in verschiedener Hinsicht neue Art von Wörterbuch entstehen; einen Eindruck davon wird man im Verlaufe der weiteren Darstellung gewinnen.

Das gewählte Darstellungsformat - zunächst in Form eines Dialogprogramms auf dem Computer - kann unterschiedlichen Interessen und Bedürfnissen genügen. Zum einen können relativ erschöpfende Informationen zur konzepturellen Struktur des entsprechenden Wortschatzbereiches gegeben werden. Im Zusammenhang damit können vorhandene Lexikalisierungstendenzen ermittelt und systematische oder zufallige

1 Die folgenden Darstellungen beziehen sich auf ein Projekt, das mit Hilfe eines Computerprogramms eine Erklärende Synonymik der kommunikativen Ausdrücke des Deutschen" erstellen will. Diese soll allerdings noch weit über die Beschreibung der Synonymiebeziehungen hinausgehende Informationen enthalten, was hier auch deutlich werden wird, und dementsprechend unter verschiedenen Gesichtspunkten nutzbar sein. Für weitere Informationen siehe Harras (1991). 
Lücken in dem untersuchten Wortfeld aufgedeckt werden. Weiterhin werden Informationen zu den synonymischen Verwendungsmöglichkeiten der entsprechenden Verben geboten. SchlieBlich sollen die Beziehungen der Situationstypen untereinander AufschluB geben über die Beziehungen, in denen einzelne Verben zueinander stehen. Auch die Erstellung eines alphabetischen Wörterbuches, das syntaktische, semantische, pragmatische und 'synonymische' Informationen $z u$ den beschriebenen Verben enthält, ist durchaus möglich und auch vorgesehen.

Im folgenden soll nun die Beschreibungssprache näher erläutert werden, die für die vorgesehenen Analysen gewählt wurde. Dabei will ich zunächst etwas zu den Situationstypen sagen, die den globalen Rahmen für die Beschreibung der kommunikativen Ausdrücke des Deutschen bilden. Dann werde ich auf die Beschreibungssprache speziellerer Ebenen innerhalb dieses Rahmens eingehen und schließlich zu den Lexikoneinträgen für die einzelnen Verben kommen, bei denen es dann allerdings weniger um Fragen der Beschreibungssprache als vielmehr um die inhaltliche Gliederung dieser Einträge geht. Damit verbunden sollen jeweils die Möglichkeiten erläutert werden, die das Dialogprogramm im einzelnen bietet.

\section{Die Rekurssituationstypen}

\subsection{Der allgemeine Rekurssituationstyp}

Die Situationstypen, von denen bereits die Rede war und die den beschreibungssprachlichen Rahmen darstellen, kommen in dem hier $z u$ erläuternden Dialogprogramm auf unterschiedlichen Hierarchiestufen vor und werden Rekurssituationstypen genannt. Diese Annahme gründet sich darauf, daß mit einer ÄuBerung, die ein Sprechaktverb enthält, auf eine (oder mehrere) andere sprachliche ÄuBerung(en) Bezug genommen wird, und zwar nicht nur auf die ursprüngliche Äußerung selbst, sondern auf die gesamte Situation, in der diese Außerung stattfand. Es ist ein Charakteristikum von Sprechaktverben, daB sie sich in spezifischer Weise auf einzelne Elemente dieser Situation beziehen. Der Terminus Rekurssituation beschreibt also lediglich eine ÄuBerungs- oder Kommunikationssituation, auf die dann wiederum in einer anderen AuBerungssituation mit sprachlichen Mitteln Bezug genommen wird. Dabei enthält der allgemeine Rekurssituationstyp diejenigen Bedingungen, die für sprachliches Handeln überhaupt erfüllt sein müssen. Hier läBt sich als invariante Basis ein Tupel herauskristallisieren, zu dessen Inventar mindestens vier Elemente gehören. Das ist zum einen S, der Sprecher, der eine ÄuBerung hervorbringt, und als zweites Element $\mathbf{H}$, der Hörer, an den diese ÄuBerung gerichtet ist. Weiterhin gehört zum allgemeinen Rekurssituationstyp das AuBerungsprodukt Sa|P|. Das ist eine Zeichenkette, die vom Sprecher 
$S$ geäußert wird. Das Äußerungsprodukt enthält zumeist einen propositionalen Gehalt $P$, auf den sich das Sprechaktverb in spezifischer Weise bezieht. Ein weiteres Element des allgemeinen Rekurssituationstyps ist schlieBlich die kommunikative Einstellung des Sprechers zum Gesagten, $\mathbf{E}(\mathbf{S})$, die er in seiner ÄuBerung zum Ausdruck bringt, denn lediglich ausgedrückte Einstellungen können hier beschrieben werden. Unter Einstellung werden allerdings auch solche Interessen verstanden, die sich ausschlieBlich auf das Herbeiführen bestimmter Zustände oder Ereignisse richten; dies trifft auf die Deklarative im allgemeinen und die rituellen Akte im besonderen zu (Beispiele hierfür sind eine Taufe vollziehen, $j m d n$. lossprechen, jmdn. freisprechen, jmdn. verurteilen) ${ }^{2}$

Dieses hier im allgemeinen Rekurssituationstyp aufgeführte Inventar ist allen lexikalischen Einheiten der Klasse der Sprechaktverben eigen und bildet damit die Grundlage jeglicher sprachlichen Äußerung, auf die mit kommunikativen Ausdrücken Bezug genommen werden kann. Der allgemeine Rekurssituationstyp stellt so etwas wie eine Bedeutungsinvariante aller Sprechaktverben dar und bildet die oberste Hierarchiestufe. Die hier benutzten Situationsrollen sind nicht zufällig gewählt; wenn man sich die Situationen und die Bedingungen klarmacht, unter denen sprachliche Kommunikation abläuft und die damit verbundenen kognitiven Prozesse in Betracht zieht, gelangt man zwangsläufig zu solchen Kategorien. Andere Untersuchungen, die sich mit der Bedeutung von Sprechaktverben befassen, kommen zu einem ganz ähnlichen Inventar, vgl. z.B. Miller/Johnson-Laird (1976), Lehmann (1976), Dirven/Goossens/Putseys/Vorlat (1982), Winkler (1986).

Es sei an dieser Stelle noch ein Exkurs zu dem Terminus Rekurssituation bzw. Rekurssituationstyp (in Anlehnung an Barwise/Perry (1987)) gestattet. Diese Terminologie mag etwas ungewöhnlich erscheinen, auch weil sie nicht so gebräuchlich ist wie die Redeweise von Originaläußerung und Wiedergabeäußerung, die sich für diesen Faktenbereich etabliert hat. Gemeint ist mit dem Begriff Rekurssituation jene

2 Mit ÄuBerungen wie

(i) Ich taufe dich auf den Namen Johannes.

(ii) Im Namen des Volkes verurteile ich Sie zu einer Freiheitsstrafe von zwei Jahren.

(iii) In zweiter Instanz wurde er freigesprochen.

werden neue Fakten in der Welt geschaffen, die zum Teil (bei verurteilen und freisprechen) auf vorangegangene tatsächliche oder vermeintliche Handlungen des Hörers Bezug nehmen. Die Einstellung des Sprechers erschöpft sich aber lediglich darin, das in der sprachlichen Formel (oder der sonstigen Āußerung) erwāhnte Faktum herbeizuführen. 
Kommunikationssituation, auf die Bezug genommen wird, wenn man in einer Äußerung ein Sprechaktverb benutzt. Es ist, wenn man so will, die Originaläußerungssituation. Situationen, die mit den gleichen Mitteln zu beschreiben sind und die gleichen oder ähnliche Elemente enthalten, werden zu Situationstypen zusammengefaßt.

Beide Terminologien - die von den Rekurssituationen und die von Original- und Wiedergabeäußerung - lassen sich zueinander in Beziehung setzen, dadurch, daB der Sprecher, der ein Sprechaktverb benutzt, immer einen Rückgriff machen muB auf die Situation, in der die reportierte Äußerung ursprünglich stattgefunden hat. Bei performativer Verwendung eines Sprechaktverbs fallt beides zusammen.

Die Termini Original- und Wiedergabeäußerung haben zunächst den Vorteil, daß sie sich selbst erklären. Die Originaläußerung ist eben jene ursprüngliche Äußerung, auf die mit der Wiedergabeäußerung Bezug genommen wird. Dabei müssen natürlich in der Wiedergabeäußerung auch die situativen Umstände der OriginaläuBerung Berücksichtigung finden, was in der Terminiologie unausgedrückt bleibt, sich beispielsweise aber in der Verbwahl niederschlagen muB. Um diesen Aspekt zum Tragen zu bringen, könnte man parallel zu den Termini Original- und Wiedergabeäußerung von Originaläußerungs- und WiedergabeäuBerungssituation sprechen. Da es hier primär um die Beschreibung von Sprechaktverben und deren Gebrauchsbedingungen geht, kommt es vor allen Dingen darauf an, die Originaläußerungssituation möglichst genau zu beschreiben. Denn nur unter Rückgriff darauf ist eine korrekte Verwendung der einzelnen Sprechaktverben möglich. Die Wiedergabeäußerungssituation spielt bei der Beschreibung der Verben eigentlich in den seltensten Fällen eine Rolle, lediglich muß man voraussetzen, $\mathrm{da} B$ die Beschreibung und Bewertung der OriginaläuBerungssituation natürlich aus der Sicht des Wiedergabesprechers erfolgt. Die Einbeziehung der Originaläußerung selbst ist insofern notwendig, als auf sie (d.h. meist auf bestimmte ihrer formalen Merkmale) und die Situation, in der sie stattfand, mit dem Verb Rekurs genommen wird, und zwar nicht auf die einzelne, ganz spezifische Situation, sondern auf Typen von Situationen. Und eben diese Bezugssituation ist mit dem Terminus Rekurssituation erfaBt.

Da es, wie wir nun gesehen haben, hauptsächlich um die Beschreibung dieser Situation geht, kann hier auf eine Dopplung der Terminologie in OriginaläuBerungs- und Wiedergabeäußerungssituation verzichtet werden. Die Beziehungen, auf die es ankommt, sollten in der Bezeichnung Rekurssituationstyp genügend deutlich werden. Dieser Terminus hat darïber hinaus noch den Vorteil, auch auf breitere Faktenbereiche 
anwendbar zu sein. So gibt es eine große Menge von Rekurssituationen, in denen keine sprachlichen Äußerungen vorkommen, auf die aber in einer (späteren) Diskurssituation Bezug genommen werden kann. So kann z.B. jemand (in einer Diskurssituation) über das letzte Fußballspiel (Rekurssituation) berichten. Die hier zu beschreibenden Rekurssituationen, in denen sprachliche ÄuBerungen stattfinden, stellen gewissermaBen eine ausgezeichnete Teilmenge aus der Menge aller Rekurssituationen dar.

\subsection{Die speziellen Rekurssituationstypen}

Eine Parametrisierung des allgemeinen Rekurssituationstyps und somit die Erzeugung spezieller Rekurssituationstypen erfolgt dadurch, daß einzelne Elemente des allgemeinen Rekurssituationstyps weiter spezifiziert und strukturiert werden. Das sind zum einen das Äußerungsprodukt und zum anderen die kommunikative Einstellung des Sprechers. Die folgenden Parameter werden auf diese beiden Elemente angewendet:

Abb.1

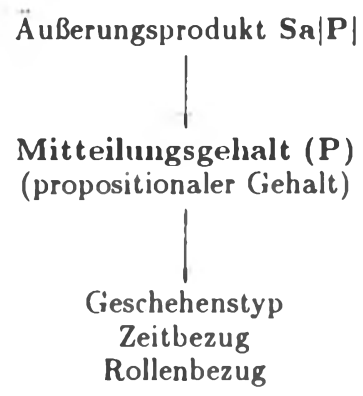


Abb. 2

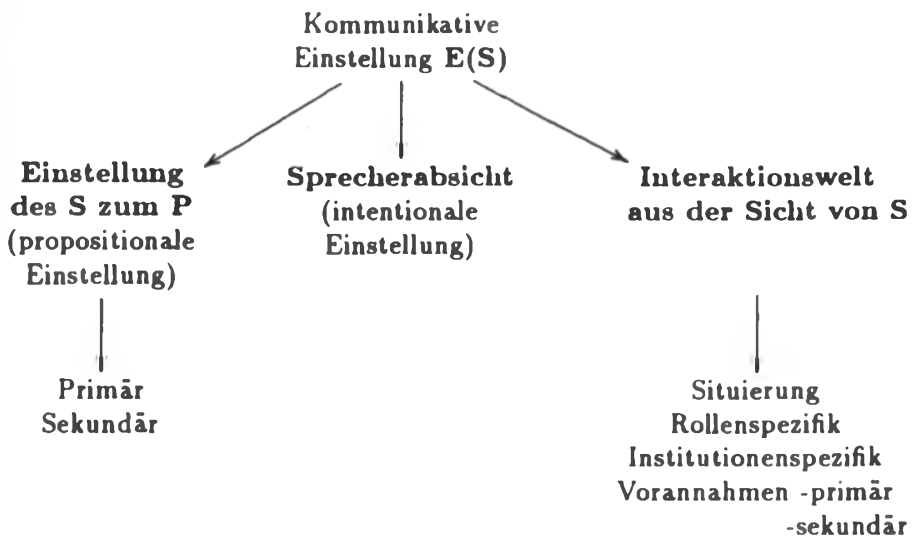

So ergibt sich eine weitere Differenzierung und Strukturierung der Sprechaktverben in bestimmte Teilmengen, die jeweils innerhalb eines speziellen Rekurssituationstyps organisiert sind. Durch die Belegung der einzelnen Elemente des speziellen Rekurssituationstyps mit unterschiedlichen Werten werden dann wiederum noch speziellere Untertypen von Rekurssituationen erzeugt, wodurch es möglich ist, den Wortschatzbereich der kommunikativen Ausdrücke hierarchisch zu strukturieren. So gibt es als Untertypen des Rekurssituationstyps der Repräsentativa beispielsweise die informellen (mit benachrichtigen, informieren, mitteilen usw.), die assertiven (mit behaupten, feststellen usw.), die sequentiellen Repräsentativa (mit argumentieren, darstellen, überzeugen usw.) und weitere Untertypen. Als Untertypen der Direktive existieren die Typen Fragen, Bitten und Aufforderungen, die zum Teil noch weiter zu differenzieren sind, was nachher am Beispiel demonstriert werden soll. ${ }^{3}$

Der Gesichtspunkt nun, unter dem das Äußerungsprodukt näher betrachtet wird, ist der des Mitteilungsgehaltes, des propositionalen Gehalts $\mathrm{P}$ der $\ddot{A} u B e r u n g$ (s. auch Abb. 1). Das Problem dabei ist, wie sich zeigen wird, daB der propositionale Gehalt sich nicht in allen Fällen auf dieselbe Art von Informationen bezieht. Die Differenzierungen des Mit-

3 Die Bezeichnung der speziellen Rekurssituationstypen erfolgte in Anlehnung an die Klassifikation der Sprechakttypen; auf die Rechtfertigung für diesen Rückgriff wird an späterer Stelle noch eingegangen. So gibt es als Haupttypen z.B. die Deklarative, die Direktive, die Repräsentative, die Expressive usw. 
teilungsgehalts - Geschehenstyp, Zeitbezug und Rollenbezug - werden mit unterschiedlichen Werten belegt. So kann man beispielsweise nur zu einer zukünftigen Handlung des Hörers auffordern, während es bei fragen egal ist, ob sich das Erfragte auf etwas Zukünftiges, Vergangenes oder Gegenwärtiges bezieht und auch ein beliebiger Sachverhalt erfragt werden kann. Loben oder tadeln kann man dagegen nur eine vergangene Handlung des Hörers, versprechen nur eine zukünftige Handlung des Sprechers usw.

Bei den Parametern, die die kommunikative Einstellung spezifizieren ( vgl. Abb. 2), die die wird unterschieden $\mathrm{zwischen}$ der propositionalen Einstellung des Sprechers zu P, E(S,P), und der Sprecherabsicht A(S), d.h. der intentionalen Einstellung des Sprechers zu H oder P. Auf Beispiele dazu komme ich noch zu sprechen. SchlieBlich gibt es noch einen dritten Aspekt, der die Interaktionswelt aus der Sicht des Sprechers betrifft und der in einem weiteren Sinne auch eine Modellierung der kommunikativen Sprechereinstellung aus dem allgemeinen Rekurssituationstyp darstellt. Die Interaktionswelt läßt sich untergliedern in die Teilbereiche kommunikative Situierung, Rollenspezifi, Institutionenspezifik und Vorannahmen des Sprechers (bezüglich des Hörers, bezüglich $P$ und seiner selbst in der entsprechenden Kommunikationssituation).

Für jedes dieser Elemente sind bestimmte Werte bereits vordefiniert, die dann mit Hilfe des Dialogprogramms einzeln aufgerufen und verschieden miteinander kombiniert werden können. Entsprechend ihrer Kombinatorik erhält man unterschiedliche spezielle Rekurssituationstypen. Ebenso ist es möglich, da $B$ der angegebenen Kombination von Werten kein Rekurssituationstyp entspricht, d.h., es existieren in einem solchen Falle natürlich auch keine Verben, die die entsprechende semantische Charakterisierung aufweisen. ${ }^{4}$ Die vordefinierten Werte sind möglichst vollständig erfaßt worden, so wie die logischen Möglichkeiten es gestatten. Diese Prozedur erfolgte zunächst noch völlig unabhängig davon, ob die einzelnen Werte bzw. Wertekombinationen tatsächlich durch ein Sprechaktverb belegt sind, d.h., ob es Lexikalisierungen für sie gibt.

Genau dort ist auch der Punkt, an dem vorhandene Lexikalisierungstendenzen ausgemacht werden können. D.h., an dieser Stelle wird klar, für

4 Auf dem Bildschirm erscheint dann der Hinweis, daB ein Rekurssituationstyp mit diesen Parameterbelegungen nicht existiert. Man hat nun die Möglichkeit, einzelne Belegungen zu ändern, bis man zu einem Rekurssituationstyp gelangt, für den auch Verben existieren. Oder man wählt nur eine einzelne Belegung aus und erhält dann alle die Rekurssituationstypen zur Auswahl, die diese Belegung enthalten und für die auch Lexikalisierungen, d.h. Sprechaktverben, vorhanden sind. 
welche Wertekombinationen Belegungen existieren, wo es Lücken gibt und, zumindest teilweise, wo die Gründe für fehlende Belegungen zu suchen sind. So kann u.a. deutlich gemacht werden, ob es sich um systematische oder zufällige Lücken handelt.

Klar ist, daß bestimmte Wertekombinationen logisch unmöglich sind (z.B. GLAUBEN und NICHT GLAUBEN). ${ }^{5}$ Das sind allerdings die wenig interessanten Fälle.

Weiterhin sind bestimmte andere Kombinationen von Wertebelegungen nicht lexikalisiert, beispielsweise kann man nicht gleichzeitig und in gleicher Weise GLAUBEN, DASS P und P ALS GUT ODER SCHLECHT bewerten. Gleiches gilt für beim Hörer zu erreichende Ziele. Vorhandene Verben wie begrüßen, gutheißen, tadeln, kritisieren präsupponieren das Bestehen des bewerteten Sachverhalts bereits. Mit ihnen kann nicht gleichzeitig das Bestehen des Sachverhalts behauptet als auch dieser Sachverhalt bewertet werden.

Oft ist auch klar, daß bestimmte Werte (häufig positiv-negativUnterscheidungen) für die Verben mit einer allgemeinen Bedeutung nicht distinktiv sind, aber durchaus zu Unterscheidungen führen, solbald die Verben eine speziellere Bedeutung haben. Diese wenigen Sätze mögen dazu genügen; weitere Beispiele lassen sich finden.

Sehen wir uns nun die einzelnen Elemente, die spezielle Rekurssituationstypen modellieren, genauer an. Der für den Mitteilungsgehalt wesentliche Aspekt ist neben dem Zeitbezug und dem Rollenbezug der Geschehenstyp, der die Art dessen charakterisiert, was mitgeteilt wird. Das können Ereignisse, Zustände, Handlungen oder aber beliebige Sachverhalte sein. ${ }^{6}$ Der Rollenbezug ist nur von Bedeutung, wenn der Geschehenstyp eine Handlung ist; dann mu $B$ auch angegeben werden, auf wen sie sich bezieht. Das kann der Sprecher sein (wie bei versprechen), der Hörer (wie bei auffordern), Sprecher \& Hörer, Dritte (Andere), oder aber der Rollenbezug ist beliebig.

5 In Versalien dargestell t werden die metasprachlichen Ausdrücke der Beschreibungssprache im Gegensatz zu objektsprachlichen Beispielen, die kursiv notiert sind.

6 Die Bezeichnung der einzelnen Geschehenstypen ist nicht einfach, da man durchaus auch 'Sachverhalt' als den allgemeinen Oberbegriff ansehen kōnnte, der den Parameter bezeichnet. Da dieser Terminus aber als eine Option zur Parameterbelegung (beliebiger Sachverhalt) nochmals auftaucht, wurde ein anderer allgeıneiner Begriff gesucht. Der Terminus 'Geschehenstyp' scheint insofern gerechtfertigt, als es ja tatsächlich der Typ des init P beschriebenen Geschehens ist, der durch ihn bezeichnet wird. 
(1) Peter versprach ihm, das Buch morgen mitzubringen.

(2) Er hat Fritz aufgefordert, sofort nach Hause zu kommen.

(3) Lisa schlägt vor, daß wir heute abend ins Kino gehen.

(4) Die Eltern haben besprochen, daß Paul in Zukunft der Großmutter beim Einkaufen helfen soll.

Der Zeitbezug des Mitteilungsgehalts kann entweder vergangen, gegenwärtig oder zukünftig sein, oder aber er spielt eigentlich keine Rolle und ist daher beliebig (wie bei den Fragen).

Die propositionalen Sprechereinstellungen umfassen sowohl epistemische als auch voluntative und evaluative Einstellungen. Es wird darüber hinaus $z$ wischen primärer und sekundärer Sprechereinstellung unterschieden, die Belegungsmöglichkeiten für beide sind allerdings die gleichen. Es soll damit lediglich die Möglichkeit geschaffen werden, hierarchische Einstellungskonstellationen festzuhalten, falls solche gegeben sind.

Die epistemische Sprechereinstellung wird in der Beschreibungssprache durch die metasprachlichen Prädikate WISSEN, GLAUBEN oder KENNEN ausgedrückt. Die ausformulierten Definitionen dieser Prädikate sind hier nach Harras (1993a und b) wiedergegeben.

Die Verwendung der beiden erstgenannten Prädikate kann Problematisches in sich bergen. Das beschreibungssprachliche WISSEN wird zunächst ähnlich wie in den üblichen Logiken definiert: S WEISS, DASS $P$ heiBt $S$ GLAUBT, DASS P \& P IST WAHR. S WEISS NICHT, DASS $P$ heiBt entsprechend S GLAUBT NICHT, DASS P \& P IST WAHR. Der zweite Bestandteil dieser Definition setzt die Wahrheit dessen, was gewuBt wird, d.h. die Erfüllungsbedingungen für den zweiten Teil sind jeweils unabhängig vom Sprecher. Das steht im Gegensatz sowohl zu den sprecherabhängigen Erfüllungsbedingungen für den jeweils ersten Teil als auch zu der hier generell gewählten Betrachtungs- und Beschreibungsweise aus Sprechersicht. Eine mögliche Lösung für das Problem besteht darin, daB man ähnlich wie Mudersbach (1984) die Wahrheitsbedingung in die Voraussetzungen einbezieht. Diese Betrachtungsweise ist dadurch gerechtfertigt, daB die Wahrheitsbedingung nicht durch die $\mathrm{Ne}$ gation tangiert wird, wie oben zu sehen war. Unter diesen Bedingungen ist WISSEN gleichzusetzen mit GLAUBEN (UNTER DER VORAUSSETZUNG DER WAHRHEIT VON P). Das kommt der Unterscheidung nahe, die in der Logik zwischen dem starken Glaubensbegriff und dem Wissensbegriff getroffen wird. Doch auch diese Unterscheidung ist an sich schon problematisch, weil es schwierig ist, unterschiedlich starke Glaubensbegriffe exakt zu bestimmen. Dabei besteht dann die Gefahr, $\mathrm{da} B$ die Definitionen ineinander übergehen und verschwimmen. Im Ver- 
lauf der weiteren Arbeit sollte ohnehin geprüft werden, ob WISSEN als Element der Beschreibungssprache tatsächlich notwendig ist. Eventuell lassen sich die vorhandenen Sprechereinstellungen mit GLAUBEN und KENNEN oder durch eine Kombination aus diesen beiden Prädikaten beschreiben. Möglicherweise kann man auch auf das Für-Wahr-Halten durch den Sprecher zurückgreifen, wie es Mudersbach (1984) in seiner epistemistischen Betrachtungsweise verwendet. Dabei wird der Standpunkt der Betrachtung immer in den Glaubenden hineinverlegt, und dessen Wissensstand ist allein der Maßstab für die Beurteilung seiner Kommunikation.

Nun zum Prädikat GLAUBEN. Es wird zunächst folgendermaßen bestimmt: S GLAUBT, DASS P heiBt: P HAT FÜR DEN SPRECHER MAXIMALE WAHRSCHEINLICHKEIT (etwa im Sinne von Kutscheras (1976) starken Glaubensbegriff). Als umgangssprachliche Umschreibung könnte man angeben: Der Sprecher ist fest von $P$ überzeugt, alle ihm bekannten Gründe sprechen für $P$ und nicht dagegen. Oder anders ausgedrückt (wie in Harras (1993b)): S H ÄLT P EHER FÜR WAHR ALS FÜR FALSCH. Entsprechend sieht es für die negierte Variante $S$ GLAUBT NICHT, DASS P aus. Erstens sollte nun überlegt werden, ob für die Beschreibung der Sprechaktverben nicht ein schwächerer Glaubensbegriff ausreichend ist, der im objektsprachlichen Bereich etwa zwischen vermuten und überzeugt sein anzusiedeln wäre. Davon unabhängig ist und bleibt ein graduierbarer Glaubensbegriff aber problematisch, weil es sowohl empirisch als auch theoretisch schwierig festzustellen und voneinander abzugrenzen ist, ob ein Sprecher stärker oder weniger stark glaubt, da $B$. Auch hier ist es darum möglicherweise günstiger, sich auf das Für-Wahr-Halten von $\mathrm{P}$ durch den Sprecher zu beschränken. Bei WISSEN käme dann gegenüber GLAUBEN noch die Voraussetzung hinzu, daB $P$ wahr ist.

Das Prädikat KENNEN soll lediglich ZUM KENNTNISSYSTEM/ KENNTNISSTAND VON S GEHÖRIC bedeuten. Das objektsprachliche Verb kennen kann eigentlich nur bei Personen, Gegenständen und ähnlichen einfachen Entitäten verwendet werden, der beschreibungssprachliche Basisausdruck KENNEN jedoch soll auch auf komplexe Sachverhalte wie Propositionen angewendet werden können. Das ist so zu verstehen, daß ein Sprecher einen Sachverhalt als Ganzens kennen kann, d.h. der Sachverhalt ist dem Sprecher zum Sprechzeitpunkt präsent, und er ist abrufbar; er gehört damit also zum aktuellen Kenntnisstand des Sprechers.

Die voluntative Sprechereinstellung wird ausgedrückt durch WOLLEN, für das folgende Festlegungen getroffen worden sind: S WILL, DASS P 
soll so verstanden werden, daß der Sprecher $P$ WÜNSCHT und P FÜR MÖGLICH BZW. REALISIERBAR hält. Entsprechend für die negative Aussage. S WILL NICHT, DASS P heißt: S WÜNSCHT P NICHT und S HÄLT P FÜR MÖGLICH BZW. REALISIERBAR. P WÜNSCHEN bedeutet, daB der Sprecher das Bestehen von $P$ dem Nicht-Bestehen von $P$ vorzieht, daB er eher $P$ als $\neg P$ anstrebt. Diese Kennzeichnung hat allerdings noch nichts mit einer evaluativen Aussage über $P$ zu tun. WOLLEN enthält also gegenüber WÜNSCHEN noch eine zusätzliche Komponente, nämlich die der möglichen Realisierbarkeit. WÜNSCHEN kann man sich dagegen auch etwas völlig Unmögliches, etwas, das nicht realisierbar ist. WÜNSCHEN wird jedoch als eigenständige propositionale Sprechereinstellung hier nicht aufgeführt, und es ist auch zu bezweifeln, ob eine solche Distinktion zur Differenzierung von Teilen des untersuchten Wortschatzbereiches oder auch von einzelnen Verben tatsächlich beitragen kann. Die genannte Spezifizierung von WOLLEN dürfte ausreichend sein.

Evaluative Sprechereinstellungen werden durch GUT bzw. SCHLECHT finden ausgedrückt, was eine positive bzw. negative Bewertung von $P$ durch den Sprecher bedeutet.

Darüber hinaus besteht die Möglichkeit, daß die propositionale Sprechereinstellung keine Rolle spielt bzw. überhaupt nicht vorhanden ist, z.B. bei bestimmten rituellen Verben, wo festgelegte Formeln verwendet werden (taufen, verurteilen usw.). Für diese Fälle ist die Belegung OHNE SPRECHEREINSTELLUNG vorgesehen. Bei der Beschreibung des allgemeinen Rekurssituationstyps wurde ja schon auf diese Besonderheit von deklarativen rituellen Akten hingewiesen.

Die vordefinierten Sprecherabsichten, d.h. die intentionalen Sprechereinstellungen, beziehen sich bis auf eine Ausnahme auf bestimmte epistemische und evaluative Einstellungen, die beim Hörer erzielt werden sollen. Die Ausnahme (S WILL, DASS Q) ist wiederum bezogen auf die eben erwähnte Gruppe der deklarativen und rituellen Sprechaktverben, die ausschließlich auf das Herbeiführen bestimmter Zustände oder Ereignisse gerichtet sind. Sie indizieren offenbar nicht im gleichen Maße kommunikative Akte wie die anderen Sprechaktverben. Q wurde hier gewählt, weil bei vielen rituellen Akten, die unter Verwendung festgelegter sprachlicher Formeln ablaufen, in denen das entsprechende Sprechaktverb geäuBert wird, in dem Sinne gar kein propositionaler Gehalt $P$ vorhanden ist. Mit dem Äußern dieser Formeln wird ein Faktum geschaffen. Das Problem bei rituellen Sprechakten ist, daB die individuelle Einstellung des Sprechers der Formel gar nicht unbedingt die des Wollens sein muß, ihm kann es z.B. völlig egal sein, ob und auf welchen Namen ein Kind getauft wird. 
Ansonsten sind die epistemischen und evaluativen Einstellungen, die beim Hörer erreicht werden sollen, die gleichen, die schon als propositionale Sprechereinstellungen auftraten. Die Option S WILL, DASS H P NICHT KENNT wurde der Vollständigkeit halber belassen, sie macht aber bei näherem Betrachten relativ wenig Sinn. Denn zweierlei kann präsupponiert werden. Als erste Präsupposition ist möglich, daB H P NICHT KENNT. Dann kann der Sprecher den Hörer ganz einfach in diesem gewünschten Zustand belassen, indem er zu P schweigt. Für diesen Fall ist dann aber kein Sprechaktverb vonnöten, und somit ist er für uns uninteressant. Auf die Unterschiede zwischen schweigen und verschwergen will ich an dieser Stelle gar nicht weiter eingehen, auch nicht auf das Paradox, daß das $\mathrm{P}$, was bei verschweigen evtl. geäußert wird, nichts mit dem oben genannten $P$ zu tun hat. Die zweite mögliche Voraussetzung ist die, daB H P KENNT. Geht man von ihr aus, wird die Sache völlig absurd. Denn mit sprachlichen Mitteln allein kann man nichts aus jemandes Kenntnissystem streichen. NICHT-WISSEN führt m.E. unter entsprechenden Voraussetzungen zu ähnlichen Problemen, die aber nicht ganz so krass in Erscheinung treten, weil WISSEN ein komplexeres Prädikat ist und mit GLAUBEN zusammenhängt.

Zwei weitere Sprecherabsichten passen nicht ganz in das Bild, denn sie betreffen keine beim Hörer zu erreichenden Einstellungen, sondern zeigen an, daB der Hörer etwas tun soll. Diese beiden Sprecherabsichten ( WILL, DASS H P TUT ODER UNTERLÄSST und S WILL, DASS H R TUT) sind für die Verben der Gruppe der Direktive vorgesehen. Und zwar die erste für die Aufforderungen und Bitten, bei denen der Hörer ja tatsächlich das tun bzw. unterlassen soll, was der propositionale Gehalt $P$ der Äußerung besagt. Die Unterscheidung $z$ wischen positiver und negativer Variante (TUN vs. UNTERLASSEN) ist hier nicht distinktiv, deswegen sind beide Varianten in diesem Falle zusammengefaBt worden. Ausnahmen, die nur die negative Alternative betreffen, wären verbieten und untersagen, die aber in ihrer Struktur ansonsten komplexer sind. Die zweite Option (S WILL, DASS H R TUT) ist den Fragen vorbehalten, bei denen sich die angestrebte Tätigkeit des Hörers auf das Beantworten der Frage beschränkt und sich nicht in gleicher Weise auf $P$ bezieht wie das Ausführen eines Befehls o.ä. Der Hörer soll ja hier nicht eigentlich $P$ tun, sondern durch das sprachliche Handeln, das sprachliche Reagieren bewirken, daß der mit $\mathrm{P}$ als für den Sprecher offen oder teilweise offen charakterisierte Sachverhalt spezifiziert wird. Aufgrund dieser doch ziemlich anders gearteten Beziehung wurde für Fragen die Sprecherabsicht S WILL, DASS H R TUT gewählt, wobei R dann eine sprachliche 
Reaktion ist, nämlich das Beantworten der Frage, und die Antwort sich in spezifischer Weise auf $\mathrm{P}$ beziehen muB. ${ }^{\text {? }}$

Bleibt noch der Bereich der Interaktionswelt aus Sprechersicht. Dieser Bereich ist am vagsten, einfach weil hier so viele außersprachliche Faktoren wie gesellschaftliche Konventionen und Organisationsbedingungen eine Rolle spielen. Sprachliche Kommunikation kann in den vielfältigsten Situationen stattfinden, die jeweils von ihnen eigenen Gesetzmäßigkeiten bestimmt werden. Dementsprechend gibt es für diesen Bereich auch nur relativ wenige vordefinierte Optionen. Es ist dagegen eher mit einer Vielzahl von Möglichkeiten zu rechnen, die nur für sehr wenige, oder gar nur für einzelne Verben und Situationen Gültigkeit haben, und die daher von Fall zu Fall gesondert angegeben werden müssen und nicht aus den vordefinierten Optionen ausgewählt werden können.

Die Interaktionswelt ist in vier herausgehobene Unteraspekte aufgegliedert. Diese beziehen sich zum einen auf die kommunikative Situierung der Äußerung, die z.B. INITIAL (wie bei fragen), REAKTIV (wie bei antworten) oder QUANTITATIV-SEQUENTIELL (wie bei diskutieren, sich unterhalten) sein kann, des weiteren auf die Rollenspezifik (PRIVAT wie bei anvertrauen, ÖFFENTLICH wie bei bekanntgeben, INSTITUTIONELL wie bei anzeigen, proklamieren, verfügen usw.), auf die Institutionenspezifik (BEHÖRDE wie bei anklagen, KIRCHE wie bei predigen, MEDIEN wie bei interviewen, moderieren u.a.) und schlieBlich auf die Vorannahmen des Sprechers, wo wieder zwischen primären und sekundären Vorannahmen differenziert wird, da man davon ausgehen muB, daB vom Sprecher häufig mehrere Vorannahmen gemacht werden, die untereinander hierarchisch strukturiert sind. Die Festlegungen, die hier für die Vorannahmen des Sprechers getroffen wurden, sind zum einen bezogen auf den propositionalen Gehalt der Äußerungen und zum anderen auf die Fähigkeiten des Hörers sowie auf bestimmte epistemische und evaluative Einstellungen des Hörers.

7 Unterschiedliche propositionale Sprechereinstellungen und Sprecherabsichten lassen sich nicht so gut an Einzelsatzbeispielen deutlich machen, deshalb wurde hier auf sie verzichtet. Größere Gruppen von Verben, für die bestimmte Absichten oder Einstellungen zutreffend sind, wurden ja jeweils genannt. AuBerdem kann das Beispiel unter Punkt 2. zur weiteren Verdeutlichung beitragen. 
1.2.1 Zusammenfassende Darstellung des Inventars der speziellen Rekurssituationstypen

(i) Propositionaler Gehalt

Geschehenstyp: Ereignis

Zustand

Handlung

beliebiger Sachverhalt

Zeitbezug: vergangen

gegenwärtig

zukünftig

beliebig

Rollenbezur: Sprecher

Hörer

Sprecher \& Hörer

Dritter (Andere)

beliebig

(ii) Provositionale Einstellung des Surechers

$S$ weiß, daß $P$

$S$ weiß nicht, $d a B P$

$S$ glaubt, daß $P$

$S$ glaubt nicht, daß $P$

$S$ kennt $P$

$S$ kennt $P$ nicht

$S$ will, daß $P$

$S$ will nicht, daß $P$

$S$ findet $P$ gut

$\mathrm{S}$ findet $\mathrm{P}$ schlecht

(iii) Intentionale Einstellung des Sprecher

$S$ will, daß H P tut oder unterläßt

$S$ will, daB H R tut

$S$ will, daß H weiß, daß $P$

$S$ will, daB $H$ nicht weiß, daß $P$

$S$ will, daB $H$ glaubt, daß $P$

$S$ will, daß $H$ nicht glaubt, daß $P$

$S$ will, daß H P kennt

$S$ will, daß H P nicht kennt

$S$ will, daB H P gut findet

$S$ will, daß H P schlecht findet

$S$ will, daß Q 
(iv) Interaktionswelt aus Sprechersicht kommunikative Situierung der ÄuBerung:

initial

reaktiv

re-reaktiv

Rollenspezifik: privat

öffentlich/institutionell

vertraut ...

Institutionenspezifik: Behörde

Kirche

Medien ...

Vorannahmen des Sprechers: ${ }^{8}$

(S nimmt an, daß) $P$ ist der Fall

$P$ ist nicht der Fall

$P$ ist im Interesse von $\mathbf{H}$

$\mathrm{P}$ ist nicht im Interesse von $\mathrm{H}$

$P$ ist im Interesse von $S$

$P$ ist nicht im Interesse von $S$

$P$ ist erwartbar

$P$ ist nicht erwartbar

$P$ ist gut

$P$ ist schlecht

$H$ weib, daB $P$

$H$ weib nicht, daB $P$

$H$ glaubt, daB $P$

$H$ glaubt nicht, daB $P$

$H$ kennt $P$

H kennt $P$ nicht

$\mathrm{H}$ findet $\mathrm{P}$ gut

$\mathrm{H}$ findet $\mathrm{P}$ schlecht

$\mathrm{H}$ ist in der Lage, $\mathrm{P}$ zu tun

$H$ ist in der Lage, $R$ zu tun

8 Die Darstellung der Vorannahmen des Sprechers ist im vorliegenden Computerprogramm - schlichtweg aus Platzgründen - vereinfacht worden. Es handelt sich natürlich stets um Annahmen, die der Sprecher macht, so daB es korrekt heißen müBte: $\mathrm{S}$ nimmt an, daB $\mathrm{P}$ der Fall ist/nicht der Fall ist, $\mathrm{S}$ nimmt an, daB $\mathrm{P}$ im Interesse/nicht im Interesse von $\mathrm{H}$ ist usw. 


\subsection{Das Zusammenspiel der Rekurssituationstypen}

Nachdem nun sowohl das Inventar des allgemeinen als auch das der speziellen Rekurssituationstypen beschrieben ist, stellt sich die Frage, wie man sich das Zusammenwirken aller dieser Faktoren vorstellen soll, welchen Stellenwert sie bei der Beschreibung der kommunikativen Ausdrücke des Deutschen haben.

Alle Komponenten repräsentieren einen (jeweils anderen) Teilbereich des Wissens über sprachliches Handeln, wobei ganz zentrale und grundlegende Elemente in den Situationsrollen des allgemeinen Rekurssituationstyps enthalten sind. Er enthält gewissermaßen die notwendigen Bedingungen für sprachliches Handeln überhaupt und spannt sich so als allgemeiner Rahmen, in den sich alle kommunikativen Ausdrücke einordnen lassen, über die gesamte Darstellung. Der allgemeine Rekurssituationstyp ist eine Abstraktion über die Verwendungen von Sprechaktverben und stellt daher so etwas wie eine Bedeutungsinvariante für diesen Wortschatzbereich dar. Er bildet als oberste Hierarchiestufe das größte gemeinsame Vielfache aller Sprechaktverben.

Innerhalb eines speziellen Rekurssituationstyps, der die nächst tiefere Hierarchiestufe bildet, ist jeweils eine Teilmenge von Sprechaktverben organisiert, die auf das darin angegebene Inventar Bezug nimmt. Die Elemente des speziellen Rekurssituationstyps stehen zum einen untereinander in Beziehung, und decken zum anderen bestimmte Bereiche bei der semantischen Beschreibung des Verbs sowie bei der Beschreibung seiner Gebrauchsbedingungen ab.

Weitere Hierarchisierungsstufen und damit noch speziellere Rekurssituationstypen ergeben sich durch die Belegung der einzelnen Elemente eines speziellen Rekurssituationstyps mit unterschiedlichen Werten. In den so entstehenden Untertypen sind dann spezifische Ausschnitte aus der zum übergeordneten Rekurssituationstyp gehörigen Verbmenge organisiert.

Die Beziehungen zwischen den einzelnen Rekurssituationstypen liegen dort völlig auf der Hand, wo es sich um Untertypen von speziellen Rekurssituationstypen handelt, so z.B. beim Verhältnis des Typs Direktive Fragen zu den Typen Direktive Prüfungsfragen, Direktive reaktive Fragen (s. dazu ausführlicher in Abschnitt 2.). Ebenso klar sind die Beziehungen 2 wischen Rekurssituationstypen, die gleichrangige Untertypen eines (speziellen) Rekurssituationstyps sind, vergleichbar etwa mit Schwesterkonstituenten im syntaktischen Sinne, wie es beispielsweise die einzelnen Typen von Repräsentativa sind. Rekurssituationstypen, die nicht Untertypen voneinander sind, stehen nur in Beziehung zueinander durch den Bezug, den sie zum allgemeinen Rekurssituationstyp haben 
sowie durch die Tatsache, da $B$ das Inventar der speziellen Rekurssituationstypen zunächst einmal gleich ist und nur die einzelnen Belegungen unterschiedlich aussehen.

Die speziellen Rekurssituationstypen mit den Belegungen ihrer einzelnen Elemente beschreiben immer nur Teilaspekte sprachlichen Wissens, die im Falle der kommunikativen Ausdrücke zu einem nicht unerheblichen Teil durch die Sprechakttheorie und ihre neueren Entwicklungen begründet sind. Das reicht von Austin (1962) und Searle (1969) über Ballmer/Brennenstuhl (1981), Searle/Vanderveken (1985) bis zu Vanderveken (1990 und 1991) und Ulkan (1992). Denn das Hintergrundwissen von Sprechakten schlieblich ist es, worauf u.a. mit Sprechaktverben Bezug genommen wird. Der Sprecher einer Äußerung, die ein Sprechaktverb enthält, bezieht sich auf relevante Aspekte der Situation, in der die sprachliche Handlung stattfindet. Dabei ist es völlig egal, ob es sich um eine performative oder eine deskriptive Verwendung des Verbs handelt. Beim performativen Verbgebrauch muß lediglich der Sprecher selbst bestimmte situative Umstände beachten, beim deskriptiven Verbgebrauch unterstellt er das dem Sprecher in der Situation, auf die er rekurriert.

Aufgrund dieses Rückgriffs auf sprechakttheoretisch fundierte Annahmen ist es nicht verwunderlich, daB die hier vorgenommene Klassifizierung der Sprechaktverben in wesentlichen Teilen mit einer Sprechaktklassifizierung übereinstimmt. Es werden darüber hinaus aber noch relevante Unterscheidungen getroffen, die für jene keine Rolle spielen. Beispielsweise ist im Rahmen einer Sprechaktklassifizierung eine Antwort nichts anderes als eine normale Behauptung, zur Antwort wird sie erst durch ihre Einbettung in eine bestimmte kommunikative Situation. Ähnlich verhält es sich bei argumentieren, das einzelne Argument ist für die Sprechakttheorie eine normale Behauptung, zum Argument in einer Argumentationskette wird es durch zusätzliche Bedingungen. Gerade diese Betrachtungsweise ist es aber, die hier favorisiert wird. Aspekte wie die eben genannten zählen zu den relevanten Informationen des lexikalischen Wissens über Sprechaktverben, das einen Teilbereich des konzeptuellen Wissens darstellt. ${ }^{9}$

Die Belegung der Sprecherabsicht beispielsweise enthält eine Reihe von handlungstheoretischen Informationen, die das Wissen um Handlungsabläufe und Zielstrukturen und -hierarchien mit einschließen. Die verschiedenen Aspekte, die den Mitteilungsgehalt der Äußerung betreffen,

9 Einen guten Überblick über die konzeptuelleın Wissen zugrunde liegenden kognitiven Strukturen erhält man in Schwarz (1992), deren Arbeit den derzeitigen Stand kognitiver Semantiktheorien gut wiederspiegelt. 
enthalten im wesentlichen Informationen über die interne Strukturierung von Propositionen. In die Beschreibung der Interaktionswelt spielt auch Weltwissen mit hinein, andererseits wird sie durch das lexikalische Wissen determiniert.

\section{Ein Beispiel für einen speziellen Rekurssituationstyp mit seinen Untertypen}

Im folgenden soll nun an einem Beispiel gezeigt werden, wie der Weg ist von der allgemeinen Rekurssituation über den speziellen Rekurssituationstyp bis hin zu seinen Untertypen und wie dabei die Belegung der einzelnen Komponenten konkret aussieht. Gegenstand dieser Betrachtungen sollen die Direktive sein.

Die hierarchische Strukturierung dieses Wortschatzbereiches soll aufgezeigt und erläutert werden. Einen ersten Eindruck davon bekommt man bereits, wenn man sich durch das Dialogprogramm einmal alle vorhandenen speziellen Rekurssituationstypen auflisten läßt. Denn sie sind so benannt, da $B$ immer die spezielleren Rekurssituationstypen dem nächst abstrakteren untergeordnet sind. So gliedern sich die Direktive zunächst in drei große Gruppen auf - Dir.frag. (Fragen), Dir.bitt. (Bitten) und Dir.aufford. (Aufforderungen) -, wobei Dir.frag. dann noch weiter untergliedert ist in Dir.frag.prüf. (Prüfungsfragen), Dir.frag.reakt. (reaktive Fragen) und Dir.frag.mass. (Fragen, die an mehrere Hörer gerichtet sind). Ähnliches ist möglich für die Aufforderungen. Diese Spezialisierung ergibt sich aus einer unterschiedlichen Wertebelegung für einzelne Elemente oder Eigenschaften.

Sehen wir uns zuerst den Rekurssituationstyp für die Direktive der Fragen-Gruppe genauer an. In dieser Gruppe sind sowohl der Geschehenstyp als auch der Zeit- und der Rollenbezug des Mitteilungsgehalts der Äußerung beliebig, d.h. sie können irgendeinen der vorgegebenen Werte annehmen, welcher Wert das ist, spielt für die Beschreibung dieser Verbgruppe keine Rolle. Der propositionale Gehalt einer Frage kann sich also sowohl auf Vergangenes als auf Gegenwärtiges oder auch auf Zukünftiges beziehen, ebenso wie er einen Zustand, ein Ereignis, eine Handlung oder einen $x$-beliebigen Sachverhalt schlechthin betreffen kann. Desgleichen ist die Rolle beliebig, auf die sich $P$ beziehen kann, es muB sich auch durchaus nicht immer um einen Bezug auf Personen handeln. Das kann man sich leicht an einigen Beispielen klarmachen. 
(5) Paul fragte seinen Vater, wann die Schlacht im Teutoburger Wald stattfand.

(6) Sie fragt ihn, ob er betrunken ist.

(7) Fritz fragt seinen Bruder, wann die Tante morgen ankommt.

Bei allen Fragehandlungen ist zunächst einmal davon auszugehen, daB sich die Einstellung des Sprechers zum propositionalen Gehalt darin manifestiert, daB der Sprecher $P$ nicht kennt (auf die Ausnahme in dieser Beziehung komme ich noch zu sprechen), da sonst die Frage sinnlos, weil unnötig wäre. Daher beabsichtigt der Sprecher, daß der Hörer $\mathbf{R}$ tut, d.h., die ihm gestellte Frage beantwortet. $\mathbf{R}$ ist eine reaktive Handlung, die sprachliche Reaktion des Antwortens, wobei sich die Antwort in spezifischer Weise auf das in der Frage geäuBerte $P$ beziehen muB, um als solche zu gelten.

Die Interaktionswelt, d.h. die Situation, in der die Frage geäuBert wird, gestaltet sich aus der Sicht des Sprechers folgendermaßen: Für die Institutionenspezifik kann es keine Festlegungen geben, da Fragen grundsätzlich nicht an bestimmte Institutionen gebunden sind, und auch bezüglich der Rollen werden keine speziellen Annahmen gemacht. Was die kommunikative Situierung betrifft, so sind Fragen in der Regel initiale sprachliche ÄuBerungen. Der Sprecher hat, wenn er eine Frage stellt, auch eine Antworterwartung; das bedeutet, er nimmt primär an, daB der Hörer $\mathrm{P}$ kennt, was zu der sekundären Vorannahme führt, daB $\mathrm{H}$ in der Lage ist, die ihm gestellte Frage zu beantworten. Würde $S$ nicht von diesen Annahmen ausgehen, wäre es sinnlos, die Frage zu stellen bzw. müBte er die Frage dann an einen anderen Hörer richten, den er für kompetent hält. Neben den genannten gäbe es noch weitere Möglichkeiten für (sekundäre) Vorannahmen, die der Sprecher machen kann. Natürlich gehört auch die Sprechereinstellung (S KENNT P NICHT) in gewisser Weise zu den Vorannahmen des Sprechers, sie könnten aber auch einfach nur $P$ betreffen (z.B. P IST INTERESSANT FÜR S, S MUSS P FÜR SEINE WEITERE ARBEIT WISSEN o.ä.). Es scheint jedoch plausibel zu sein, daB die oben genannte Art und Reihenfolge der Vorannahmen zunächst einmal für alle Arten von Fragen Gültigkeit besitzt.

An dieser Stelle sei gesagt, daB die Angaben zu den Vorannahmen des Sprechers ohnehin auf eine ziemlich verschachtelte Weise zustande kommen. Es handelt sich immer um Hypothesen des Sprechers, der das entsprechende Verb benutzt, darüber, welche Annahmen der Sprecher gehabt haben muB bzw. kann, der die ÄuBerung in der Rekurssituation produziert hat, damit sie als Frage oder zumindest Frageversuch eingestuft werden kann. Das ist auch der Grund dafür, warum sich bei den 
Vorannahmen nur Angaben über das Wissen des Hörers, nicht aber über das Wissen des Sprechers finden.

Soweit die Charakteristika, die für alle Direktive der Fragen-Gruppe gelten. Neben diesen allgemeinen Frageverben lassen sich noch einige spezielle Gruppen herauskristallisieren, die Untertypen des Rekurssituationstyps Dir.frag. bilden. Das sind z.B. die Verben für Fragen, die reaktiv gebraucht werden, wie nachfragen, rückfragen, zurückfragen.

(8) Er fragte noch einmal beim Finanzamt nach.

(9) Da muß ich gleich nochmal zurückfragen, wie Sie diesen Terminus hier verstehen. (als Gegenfrage statt der Antwort in einer Diskussion geäuBert)

Diese Verben haben die gleichen Parameterbelegungen wie die übrigen Fragen-Verben, lediglich die kommunikative Situierung ist als reaktiv eingestuft. Sie bezeichnen immer Fragen, die eine Reaktion auf eine vorangegangene Äußerung oder Frage darstellen. Eine zweite Untergruppe von Verben charakterisiert Fragen, die sich grundsätzlich an mehrere Hörer richten, (die Gruppe Dir.frag.mass.). Zu dieser Gruppe gehören herumfragen, rumfragen, rundfragen. Hier wird in der Regel mehreren Personen die gleiche oder eine ähnliche Frage gestellt.

(10) Ich werde mal rumfragen, wer am Sonnlag alles kommen kann.

(11) Der Schaffner fragte im Ableil herum, ob jemand fünfzig Mark wechseln könne.

Auch in dieser Gruppe ist nur ein Wert anders belegt als bei dem allgemeineren Typ Dir.frag., nämlich die Rollenspezifik in der Interaktionswelt aus Sprechersicht. Sie besagt, daß die Frage an mehrere Hörer gerichtet ist. Ein dritter Typ von speziellen Fragen-Verben sind die für die Prüfungsfragen (sie stellen auch die vorhin angekündigte Ausnahme dar). Die propositionale Sprechereinstellung ist hier nämlich nicht die des Nicht-Kennens. Im Falle der Prüfungsfragen kennt der Sprecher P; das ist notwendig, damit der $Z$ weck dieser Fragen erfüllt werden kann, der darin besteht, daB der Sprecher das Wissen des Hörers bezüglich $P$ überprüft. Dazu muB der Sprecher $P$ kennen, zumindest aber muB ihm $P$ in irgendeiner Weise vorliegen (Vokabelliste o.ä.). In diese Gruppe gehören beispielsweise die Verben abfragen und examinieren. AuBer durch die propositionale Sprechereinstellung unterscheiden sie sich noch durch eine festgelegte Rollenspezifik von den allgemeinen Fragen-Verben. Der Hörer ist hier Lernender, der Sprecher Prüfender (beides kann durchaus in einem weiten Sinne verstanden werden). 
(12) Professor Meyer war bekannt dafür, daß er die Studenten besonders gründlich examinierte.

(13) Die Freundinnen fragen sich gegenseitig die Englisch-Vokabeln ab.

Aus dieser Darstellung sollte noch einmal die hierarchische Ordnung des untersuchten Wortschatzbereiches deutlich geworden sein. Die Direktive gliedern sich auf in drei verschiedene spezielle Rekurssituationstypen, u.a. die Fragen. Diese haben nochmals drei Untertypen, bei denen einzelne Parameter anders belegt sind als beim Typ Dir.frag. und die jeweils eine Teilmenge der Verben umfassen, die in Dir.frag. enthalten sind.

Sehen wir uns zum Vergleich die zwei anderen speziellen Rekurssituationstypen der Direktive an, die Bitten und die Aufforderungen. Sie haben viele Gemeinsamkeiten, im Grunde genommen könnte man die Bitten auch als höfliche Form von Aufforderungen klassifizieren. ${ }^{10}$ Der propositionale Gehalt der ÄuBerung bezieht sich bei beiden Typen auf eine zukünftige Handlung des Hörers. Die propositionale Einstellung des Sprechers äuBert sich darin, daB er will, daß P eintritt. Der Sprecher hat die Absicht, den Hörer zum Ausführen oder Unterlassen von ebendiesem $\mathrm{P}$ zu bewegen. Die Unterscheidung $\mathrm{zwischen} \mathrm{P}$ tun oder $\mathrm{P}$ unterlassen ist in diesem Falle nicht distinktiv. Allenfalls könnte man als Belegung für S WILL, DASS H P UNTERLÄSST die Verben verbieten oder untersagen anführen. Ansonsten können aber alle Verben dieser beiden Gruppen sowohl zum Ausführen einer Handlung als auch zur Unterlassung einer Handlung auffordern. Das ist der Grund dafür, warum in diesem Fall die negierte Variante der Belegung der Sprecherabsicht nicht gesondert aufgeführt wurde. Wenn sich der Sprecher mit der Aufforderung oder der Bitte an den Hörer wendet, dann nimmt er natürlich auch an, $\mathrm{da} B$ der Hörer in der Lage ist, $\mathrm{P}$ zu tun (bzw. zu unterlassen). Bitten und Aufforderungen sind ebenso wie Fragen initiale Sprechakte, die in der Regel (auBer bei ganz speziellen Verben) keine Institutionenspezifik aufweisen. Bei der Rollenspezifik innerhalb der Interaktionswelt könnte man, je nachdem, ob man diese Art von Angaben noch darunter fassen will, vermerken, daB Bitten vom Sprecher sehr höflich formulierte ÄuBerungen sind, während das bei Aufforderungen nicht unbedingt der Fall zu sein braucht, und daB bei Nichtbefolgung einer Aufforderung eher Sanktionen angedroht werden können, als wenn jemand einer Bitte nicht nachkommt.

10 In der Tat gibt es etliche Verben, bei denen die Zuordnung entweder zu den Bitten oder zu den Aufforderungen schwerfält, weil sie je nach Situation zur Beschreibung beider Sprechhandlungen benutzt werden kōnnen. Das sind eher periphere direktive Verben wie etw. anregen, jmdn. ansprechen (weil er etwas tun soll), jmidm. anraten etw. zu tun, schlauchen. 
Zumindest bei den Aufforderungen ließe sich nun noch eine weitere Differenzierung und Strukturierung vornehmen, indem man weitere Subtypen schafft, die spezifische Parameterbelegungen haben und sich darin vom speziellen Rekurssituationstyp Dir.aufford. unterscheiden. Infrage kommen beispielsweise die Befehle, die ein Unterordnungsverhältnis voraussetzen und teilweise an bestimmte Institutionen gebunden sind. Die Möglichkeit der Verhängung von Sanktionen ist bei ihnen am stärksten gegeben. Ähnlich verhalten sich Weisungen. Bei Anleitungen und Ratschlägen sind im Gegensatz dazu kaum Sanktionsandrohungen möglich, sie sind nicht an Institutionen gebunden und setzen auch kein Unterordnungsverhältnis der Gesprächspartner voraus. Wie bereits angedeutet, ließe sich auch ein spezieller Untertyp aufmachen, der zur Unterlassung von $\mathrm{P}$ auffordert, also die Verbote. Weitere Anregungen gerade zu dieser Gruppe von Verben kann man sich bei Hindelang (1978) holen. Dabei ist allerdings immer $z u$ beachten, daB sich Untertypen von speziellen Rekurssituationstypen nur dort einführen lassen, wo es tatsächlich spezielle Lexikalisierungen, d.h. Verben, gibt. Das Vorhandensein einer spezifischen Sprechhandlung ist dafür noch nicht ausreichend, da auf sie häufig nur mit einem relativ allgemeinen Verb Bezug genommen wird. So kann z.B. auf ganz spezielle Fragehandlungen wie Regiefragen, verschiedene Arten von rhetorischen Fragen u.a. zwar mit dem Verb fragen Bezug genommen werden, es existiert aber kein eigens für diese Fälle vorgesehenes Verb. Uns geht es aber in erster Linie um eine Beschreibung und Klassifizierung der Sprechaktverben, und nur in Verbindung damit kommen die zugehörigen Sprechhandlungen zur Sprache.

Ähnliche Spezifizierungen und Differenzierungen von Rekurssituationstypen wie bei den Direktiven finden sich auch bei anderen Teilen des hier untersuchten Wortschatzbereiches. So umfassen beispielsweise die Repräsentativa vorläufig zehn Untertypen, z.B. die informellen Repräsentativa, die assertiven Repräsentativa, die sequentiellen, öffentlichen, vertraulichen und zukïnftigen Repräsentativa sowie verschiedene Gruppen von reaktiven Repräsentativa.

Ausgehend von den speziellen Rekurssituationstypen bzw. deren Subtypen kann man sich nun die Verbmenge ansehen, die zu dem jeweiligen Typ gehört. Aus dieser Verbmenge kann man einzelne Verben markieren und sich von dem Programm den vollständigen Lexikoneintrag für das Verb zeigen lassen.

\section{Die Lexikoneinträge}

Die Lexikoneinträge für die einzelnen Verben enthalten folgende Angaben: syntaktische Umgebung, Bedeutung, Verwendungsspezifik, Feldzu- 
gehörigkeit, mögliche Synonyme, mögliche Antonyme, Kommentar und schlieBlich Belege.

In der Formulierung der syntaktischen Umgebung und der Bedeutungsangabe wird ganz explizit der Bezug zu den Rekurssituationstypen hergestellt, indem angegeben wird, welche dort enthaltenen Elemente die einzelnen Argumente repräsentieren.

Das Syntaxfeld enthält zunächst eine an die Umgangssprache angelehnte Angabe der möglichen Argumente des Verbs in der im Satz auftretenden Reihenfolge. Anschließend wird in einer Art sehr stark vereinfachtem Subkategorisierungsmerkmal dargestellt, in welcher Form die einzelnen Argumente auftreten können. Für diese Darstellung wird eine verkürzte Nebensatzstruktur (d.h. mit dem Verb in Endstellung) gewählt, die mehrere Möglichkeiten der Vereinfachung bietet. Zum einen muB das Subjekt hier nicht mehr aufgeführt werden, da es in der Regel immer an derselben Stelle in Form einer Nominalphrase im Nominativ vorkommt. Auftretende Besonderheiten werden natürlich gesondert angegeben. Zum zweiten erscheinen Verben mit abtrennbarem Präfix bei dieser Art der Darstellung stets ungetrennt, und ein evtl. vorkommendes Reflexivpronomen steht immer an erster Stelle und kann dort problemlos in Form eines Merkmals notiert werden. Aufgrund der gewählten Schreibweise bezieht sich das erste der aufgeführten Argumente in der Regel auf $\mathrm{H}$, das $\mathbf{z w e i t e}$ auf $\mathrm{P}$. Ausnahmen werden auch hier jeweils angegeben. Ansonsten hält sich die Darstellung an übliche Notationen. Fakultative Argumente werden in runden Klammern angegeben, Alternativen durch Schrägstriche voneinander abgetrennt, Kasusangaben stehen in spitzen Klammern. Die verschiedenen Formen von Korrelaten und sogenannten nominalen Einbettungsstützen werden gesondert aufgeführt. ${ }^{11}$ Im AnschluB an diesen Eintrag erfolgen Angaben, die Passivierbarkeit, unpersönlichen Gebrauch, Anschluß mit der direkten Rede usw. betreffen, jeweils in Form von Merkmalen. Für die Eintragungen zur syntaktischen Umgebung existiert darüber hinaus eine Hilfsfunktion, mittels deren man sich zeigen lassen kann, was die einzelnen Angaben bedeuten.

Ähnlich strukturiert ist das semantische Feld. Hier erfolgt zunächst eine lexikographische Paraphrase der Verbbedeutung, woran sich eine detailliertere Bedeutungsbeschreibung anschlieBt, die den Bezug herstellt zu den Elementen des allgemeinen und speziellen Rekurssituati-

11 An dieser Stelle ist auch Platz vorgesehen für die Eintragung markanter modaler Merkmale (mündlich, schriftlich, telefonisch, ausführlich usw.) u.ä. Besondere Formen kōnnen gesondert angegeben werden, wenn beispielsweise ein Verb vorwiegend als Partizip oder im Infinitiv auftritt. 
onstyps, zu dem das Verb gehört. Auf eine durchgängig formale Beschreibung der einzelnen Bedeutungsbestandteile wird hier verzichtet, da diese sehr komplex ausfallen müBte und dadurch die Lesbarkeit erheblich einschränken würde. Zu überlegen wäre jedoch, ob noch Angaben, die die für einzelne Verben notwendigen Präsuppositionen betreffen, als ein zusätzlicher Punkt in diese Bedeutungsbeschreibung mit aufgenommen werden sollten, da es auch Verben gibt, die sich nur darin voneinander unterscheiden.

Die sich daran anschließenden Angaben zur Verwendungsspezifik erläutern die Situationen genauer, in denen das entsprechende Verb verwendet wird, geben spezifischere Bedingungen, Konnotationen und Ausnahmen an und sind in dieser Hinsicht viel expliziter als das im speziellen Rekurssituationstyp oder auch in der Bedeutungsangabe möglich ist. Hier ist auch der Ort, an dem Erläuterungen zu Verwendungsweisen von Verben, die Bezug nehmen auf ganz spezifische Arten von sprachlichen Handlungen, ihren Platz finden. So können an dieser Stelle sehr feine Bedeutungsnuancen und -unterschiede erläutert werden, die oft nicht direkt von dem in den Rekurssituationstypen enthaltenen Inventar abhängen und von daher im Feld für die Bedeutungsangabe keinen Platz finden und erst bei der Verwendungsspezifik aufgelistet werden können. Im großen und ganzen ist dieses Feld aber der Beschreibung typischer Gebrauchsbedingungen und Verwendungsweisen von Verben vorbehalten.

Es folgen die Angaben zur Feldzugehörigkeit der Verben. In ihnen spiegelt sich noch einmal ganz klar die hierarchische Strukturierung des untersuchten Wortschatzbereiches wider.

Anschließend erfolgen Eintragungen zur Synonymie und zur Antonymie. Bei den Synonymen wird zwischen starken und schwachen unterschieden, beides ist wiederum in einem Erklärungsfeld erläutert. ${ }^{12}$ Die schwachen Synonyme umfassen im Prinzip alle Verben eines speziellen Rekurssituationstyps, da sie im Hinblick auf die Eigenschaften, die diesen Rekurssituationstyp konstituieren, ausgewählt werden. Bei starken Synonymen müssen noch zusätzliche Eigenschaften hinzukommen.

Genauere Erläuterungen dazu finden sich jeweils im Kommentar, der speziell den synonymischen Eigenschaften gewidmet ist, die hier als Verwendungseigenschaften der Verben betrachtet werden. Um das deutlich zu machen, werden die Verben in typische schwach bzw. stark synonymische Kontexte eingesetzt, und die Grammatikalität der Sätze bzw.

12 Umfangreiche Erläuterungen zur Synonymieauffassung, wie sie hier vertreten wird, finden sich in Harras (erscheint). 
die Bedeutungsgleichheit in vergleichbaren Situationen wird überprüft. Die Ergebnisse dieser Ersetzungen werden kommentiert und mögliche Erklärungen aufgezeigt. Auf diese Weise soll versucht werden, die in einer theoretischen Beschreibung der Synonymiebeziehungen gefundenen Kriterien an den Beziehungen $z$ wischen einzelnen Verben wieder auffindbar zu machen und so die theoretische Synonymiebeschreibung transparent werden zu lassen. Dabei darf Synonymie weder ausschließlich auf semantische Gleichheit noch auf gleiche syntaktische Umgebungen reduziert werden. Es ist jeweils die spezifische Hinsicht herauszuarbeiten, unter der $z$ wei Verben bedeutungsgleich und im entsprechenden Kontext durch einander ersetzbar sind.

Als letztes erscheinen die Belege, die aus verschiedenen Korpora so ausgewählt wurden, daß sie das Verb in seinen verschiedenen syntaktischen Umgebungen zeigen, daB Verwendungsspezifika und spezielle Bedeutungsvarianten zum Ausdruck gebracht werden.

\section{Fazit}

Anhand der vorangegangenen Darlegungen sollte klar geworden sein, wie eine systematische Beschreibung der Eigenschaften sowie der Verwendungsbedingungen lexikalischer Einheiten aussehen könnte. Dabei ist das vorgestellte Dialogprogramm auf Grund seines Formats und seiner technischen Möglichkeiten sicher auch mit gewissen Einschränkungen verbunden (einige Beispiele dafür wurden ja an den entsprechenden Stellen genannt), andererseits kann eine solche Vorgehensweise aber auch zu anderen Betrachtungsweisen führen und neue Diskussionsmöglichkeiten eröffnen.

An dieser Stelle seien noch einmal kurz die drei unterschiedlichen $\mathrm{Zu}$ griffsmöglichkeiten erläutert, die man auf dieses Programm hat. Ein Zugriff kann über die Namen für die speziellen Rekurssituationstypen erfolgen. Dann erhält man vom Programm alle zu einem Typ gehörigen Parameter, die Menge der Sprechaktverben sowie die ihnen zugeordneten Lexikoneinträge. Ein weiterer Zugriff ist über einzelne Parameterbelegungen möglich. Die eingegebenen Werte werden speziellen Rekurssituationstypen zugeordnet, und das Programm liefert die gleiche Menge an Informationen wie schon bei der erstgenannten Zugriffsmöglichkeit. Die zusätzlichen Informationen, die man durch diese Art des Zugriffs bekommt, bestehen darin, daß man erfährt, für welche Parameterbelegungen keine speziellen Rekurssituationstypen und somit auch keine Sprechaktverben existieren und in welchen unterschiedlichen Rekurssituationstypen einzelne Parameterbelegungen vorkommen können. Die dritte Zugriffsmöglichkeit ist die über das einzelne Verb. Hierbei gibt man 
ein bestimmtes Verb ein, über das man nähere Informationen wünscht, und erhält dann den vollständigen Lexikoneintrag. Von dort aus ist auch ein Rückgriff auf die übrigen Verben dieses Rekurssituationstyps möglich ebenso wie auf die einzelnen Parameter des Typs. Diese Art des Zugriffs ermöglicht es, sich gezielt bestimmte Informationen über ein einzelnes Verb zu verschaffen, ohne erst das gesamte System durchlaufen zu müssen. Bei weitergehendem Interesse ist ein Zugang aber ohne weiteres realisierbar.

Schon aus der Art der verschiedenen Möglichkeiten des Zugriffs auf die Daten wird ersichtlich, daB diese Darstellungsform ganz unterschiedlichen Erkenntnisinteressen dienen kann. Das ist ein Vorteil des Systems. Hier kann sich sowohl der an der Verwendung des einzelnen Verbs Interessierte Auskunft holen als auch derjenige, dem es auf die Erklärung der Zusammenhänge innerhalb eines bestimmten Wortschatzbereiches ankommt. So ist das System vielseitig anwendbar und die Möglichkeiten gehen weit über die Nutzung als Wörterliste bei Formulierungsschwierigkeiten hinaus.

Es wird versucht, die Beziehungen $\mathbf{z w i s c h e n ~ S p r e c h a k t v e r b e n ~ u n d ~}$ Sprechakten darzustellen. Bei der Beschreibung der einzelnen Verben wird konsequent auf die ihnen zugrunde liegenden konzeptuellen Strukturen zurückgegriffen sowie auf ihre Einbettung in Typen von Situationen. Auf diese Weise entsteht eine Art von Gebrauchssemantik. In einem späteren Arbeitsschritt könnte man versuchen, die nicht durch Sprechaktverben abgedeckten sprachlichen Handlungen in Beziehung zu setzen zu dem hier dargestellten System der kommunikativen Ausdrücke.

\section{Literatur:}

Austin, John L. (1962): How to Do Things with Words. Oxford.

Ballmer, Thomas T./Brennenstuhl, Waltraud (1981): Speech Act Classification. Berlin/Heidelberg/New York. (Language and Communication 8).

Barwise, Jon/Perry, John (1987): Situationen und Einstellungen. Berlin/New York. (Studienbuch Grundlagen der Kommunikation).

Dirven, René/Goossens, Louis/Putseys, Yvan/Vorlat, Emma (1982): The Scene of Linguistic Action and its Perspectivization by Speak, Talk, Say and Tell. Amsterdam/Philadelphia. (Pragmatics \& Beyond III:6).

Harras, Gisela (1991): Konzeption zu einer erklärenden Synonymik kommunikativer Ausdrücke des Deutschen. Ms. Mannheim - (1993a): Manuskript zu einem Vortrag ūber ESKA im IdS-Kolloquium. Ms. Mannheim.

- (1993b): Erläuterungen zum Teilprojekt: Tendenzen der Lexikalisierung kommunikativer Handlungsspielräume, besser: Situationstypen und lexikalische Konzepte. (Papier für den SFB 245). Ms. Mannheim. - (erscheint): Synonymik und Synonymie. Tübingen. 
Hindelang, Gōtz (1978): Auffordern. Göppingen. (Göppinger Arbeiten zur Germanistik 247).

Kutschera, Franz von (1976): Einfūhrung in die intensionale Semantik. Berlin/New York. (Studienbuch Grundlagen der Kommunikation).

Lehmann, Dorothea (1976): Untersuchungen zur Bezeichnung der Sprechaktreferenz im Englischen. Bern/Frankfurt/M./München. (Forum Linguisticum 8).

Miller, George A./Johnson-Laird, Philip N. (1976): Language and Perception. Cambridge, Mass.

Mudersbach, Klaus (1984): Kommunikation über Glaubensinhalte. Berlin/New York. (Grundlagen der Kommunikation).

Schwarz, Monika (1992): Kognitive Semantiktheorie und neuropsychologische Realitāt. Tübingen. (Linguistische Arbeiten 273).

Searle, John R. (1969): Speech Acts. Cambridge.

Searle, John R./Vanderveken, Daniel (1985): Foundations of Illocutionary Logic. Cambridge.

Ulkan, Maria (1992): Zur Klassifikation von Sprechakten. Tübingen. (Linguistische Arbeiten 174).

Vanderveken, Daniel (1990 und 1991): Meaning and Speech Acts. 2 vols. Vol. 1: Principles of Language Use.

Vol. 2: Formal Semantics of Success and Satisfaction. Cambridge.

Winkler, Edeltraud (1986): Syntaktische und semantische Eigenschaften von verba dicendi. Diss.(masch.). Berlin. 